\title{
Image Classification Using Fusion of Holistic Visual Descriptions
}

\author{
Padmavati Shrivastava \\ Dr. C.V. Raman University, Bilaspur, 495113, Chhattisgarh, India \\ Email: padmavati.shrivastava@yahoo.co.in
}

Dr. K. K. Bhoyar

Yeshwantrao Chavan College of Engineering Nagpur, 441110, Maharashtra, India

\section{Dr. A.S. Zadgaonkar}

Former Vice Chancellor, Dr. C.V. Raman University, Bilaspur, 495113, Chhattisgarh, India

\begin{abstract}
An efficient approach for scene classification is necessary for automatically labeling an image as well as for retrieval of desired images from large scale repositories. In this paper machine learning and computer vision techniques have been applied for scene classification. The system is based on feature fusion method with holistic visual color, texture and edge descriptors. Color moments, Color Coherence Vector, Color Auto Correlogram, GLCM, Daubechies Wavelets, Gabor filters and MPEG-7 Edge Direction Histogram have been used in the proposed system to find the best combination of features for this problem. Two state-ofthe-art soft computing machine learning techniques: Support vector machine (SVM) and Artificial Neural Networks have been used to classify scene images into meaningful categories. The benchmarked Oliva-Torralba dataset has been used in this research. We report satisfactory categorization performances on a large data set of eight categories of 2688 complex, natural and urban scenes. Using a set of exhaustive experiments our proposed system has achieved classification accuracy as high as $92.5 \%$ for natural scenes (OT4) and as high as $86.4 \%$ for mixed scene categories (OT8). We also evaluate the system performance by predictive accuracy measures namely sensitivity, specificity, F-score and kappa statistic.
\end{abstract}

Index Terms-Scene Classification, Feature Fusion, Image Mining, Low-level features, Kappa Statistic.

\section{INTRODUCTION}

In the recent years, advancements in technology have made procurement of multimedia content easier than ever before. The use of World Wide Web has made it possible to exchange acquired content throughout the world, thus multiplying the amount of data available and accessible. Multimedia data consists of various media types such as text, images, audio, video sequences and animation. Advanced and readily available methods of image acquisition have led to the creation of a variety of digital images such as of a physical scene or the interior tissue structure of an organ. This implausible growth results in the generation of very large and detailed image databases which necessitates the development of intelligent systems to understand such large, complex, information-rich data sets. Computer vision is a field of study which combines different methods for acquiring, processing, analyzing and understanding images. Image mining is a heterogeneous research area which embraces a large spectrum of methods from computer vision, image processing, image acquisition, image retrieval, data mining, and machine learning. It essentially deals with extracting inherent patterns from collection of images [1].

Content based image mining consists of various data mining tasks which exploit image content based features to discover knowledge from a collection of images. The outcome of different mining tasks (classification, clustering or generation of association rules) may vary from one application domain to another. For example in [13] a study of area of land use and land cover change analysis based on satellite images is presented. In the domain of medical diagnosis an image mining system may utilize the results of clinical reports (textual data) along with the content of images in the form of X-ray or CT scan image to predict a disease. Similarly, images can be clustered into groups based on their content such as similar color or shape which can find application in content based image retrieval.

This paper addresses the task of scene understanding and development of a system which can efficiently perceive, process, and understand visual data in scene images. The aim of our work is efficient classification of scene images into appropriate category using machine learning and computer vision techniques. Regarding scene perception two types of cognitive approaches are used. The first is an object-centered approach which describes a scene in terms of the objects contained in it and the second is the scene-centered approach which does not rely on the occurrence of objects.

Our work is essentially scene centered and the purpose is to provide a "holistic" description of a scene using low level visual cues and assign it to its category such as 
sunrise/sunset, beach, highway, waterfalls or to be a basic block in computer vision based expert systems. We have chosen this approach since it closely resembles human perception of scenes. A quick visual perception does not heavily rely on the recognition of objects and detailed information. The presence of certain kind of objects is deduced even though it is not necessary that each object is recognized. The global image features can be used to assess the composition of a scene thus serving as an aid to rapid scene recognition.

Scene classification is useful in applications such as content-based image organization and context-sensitive management of images. The primary contributions of this paper are two-fold. Firstly to evaluate how the global features could contribute to real-world scene categorization, we extract appropriate low-level features and use early fusion to find the best combination of stateof-the-art computer vision features. Secondly, we analyze two different classifiers using cross-validation procedures for scene classification.

The rest of the paper is organized as follows: Section 2 presents the motivation behind present work. Section 3 gives a brief overview of previous work in scene categorization using low level descriptions. Section 4 presents the different kinds of image descriptors used in this paper as well as description of the data set used. It also elaborates the feature fusion technique and classifiers used. Section 5 presents experimental results. Section 6 presents detailed result analysis. Section 7 presents the advantages of our approach. Finally, Section 8 draws the conclusions and future directions.

\section{Motivation}

With advancements in technology the multitude of images generated every day necessitates their categorization, organization and retrieval in a fast and efficient way. For example a family on a vacation trip may capture images of coastal areas, skyscrapers, mountains and waterfalls. Automatic categorization of such scenes would help in effective management and access. Thus scene classification into semantic categories (e.g. coast, mountains and streets), is a challenging problem nowadays. The major approaches to scene classification are based on low level-features or semantic features. The motivation behind our work is find the answer to the question "Is it possible to capture the gist of the scene using a low-dimensional signature (feature) vector?"Although there is semantic gap between lowlevel features and high- level concepts, but effective lowlevel discriminating features which provide holistic scene understanding information may be used to derive middle level and symbolic and/or semantic features.

\section{LITERATURE REVIEW}

Psychophysical and psychological studies have shown that scene identification by humans can proceed, in certain cases, without any kind of object identification suggesting the possibility of coarse scene identification from global low-level features [11], [12]. A number of research studies have attempted to derive high level image semantics from low level features. This section presents notable contributions in this area. Szummer and Picard [9] and Yiu [10] propose algorithms for indoor outdoor scene classification. Yiu uses color and dominant directions for image classification over a dataset of 500 images. Szummer and Picard have proposed color and texture features extracted over whole image as well as over $4 \times 4$ sub-blocks of images to perform classification over a dataset of 1343 images which depict typical family and vacation scenes including snow, bright sun, sea, sunset, night and, silhouette scenes. Initial contributions in the area of scene classification can be found in the works of Paek et al. [26], Savakis and Luo [27], GuerinDugue and Oliva [28] and N. Serrano, A. Savakis, J. Luo [29].

In [8] Vailaya et.al have considered the hierarchical classification of vacation images on a database of 6931 vacation photographs. For indoor/outdoor classification 10x10 sub-block color moments in LUV space; for city/landscape edge direction histograms and coherence vectors; for sunrise/sunset/mountain spatial moments, color histograms and coherence vectors in HSV/LUV space have been used. In [13] authors manually label each training image with a semantic label and train $\mathrm{k}$ classifiers (one for each semantic label) using support vector machines (SVM). Each test image is classified by the $\mathrm{k}$ classifiers and assigned a confidence score for the label that each classifier is attempting to predict. A k-nary label-vector consisting of k-class membership is generated for each image. The system is tested on 15 different scene categories. In the work of Oliva and Torralba [15] the scene structure is estimated by the means of global image features. The scene is described holistically by their degree of naturalness, openness ruggedness, expansiveness, etc. Spatial envelope representation using DST has been used to evaluate the spatial layout properties. The approach in [16] is used to classify and organize real-world scenes along broad semantic axes. Firstly, all the scenes are classified according to an Artificial to Natural axis. Then, natural scenes are organized along the Open to Closed axis whereas artificial environments are classified according to the Expanded to Enclosed scene's axis. In [17] the authors represent the image of a scene by a collection of local regions, denoted as code words obtained by unsupervised learning. Each region is represented as part of a "theme". The categorization is performed on a large set of 13 categories of complex scenes. Bosch et al. [18] use a probabilistic model to recognize the objects and classify the scene based on these object occurrences.

Several approaches using low level descriptors have been studied for scene classification. The authors in [20] describe scene images through multimodal features and explore the complementary characteristics of these features. A user labeling procedure has been introduced to reduce semantic gap. In [21] a multi-scale, statistical approach for representing images aimed at scene 
categorization is presented. At different levels, sets of features that represent exclusively the scene are selected. The non-characteristic elements such as foreground objects which do not contribute to scene classification are disregarded. Authors have obtained good results even with simple features like local color image histograms. In [22] authors have developed ARTSCENE: a neural system for classifying scenes. They assume that boundary, surface, and spatial information is combined to represent scene gist. The gist of a scene is represented by its spatial layout of colors and orientations. In our approach we aim at evaluating most discriminating color, texture features along with edge descriptors using two state of the art classifiers SVM and Neural Networks (NN). Features have been fused to find the best combination and classifier evaluation has been done using an exhaustive set of experiments; the SVM parameters have been derived using 10 fold cross validation.

\section{MATERIALS AND METHODS}

Any scene classification system must extract suitable features and use some learning mechanism to classify test or input images. This section outlines the individual features, the classifiers and dataset used in our experiments.

\section{A. Image Descriptors}

Feature extraction from images and selection of appropriate features is the key to the success of any image mining task [24]. The distinguishing low-level image features used in this paper are color, texture and edges.

\section{Color}

Human eye is more sensitive to color; therefore color acts as a discriminative feature for scene understanding applications particularly those which include outdoor images [23]. Color feature is robust to background complication and is independent of image size and orientation. For example a mountain scene can be characterized by blue sky on the top whereas a forest scene will contain large portions of green shades. The color features used are:

\section{Color Moments}

Color moments are scaling and rotation invariant and are based on the assumption that the color distribution in an image can be interpreted as a probability distribution. The moments of this distribution can then be used as features to identify that image based on color. In this work three lower order color moments Mean, Standard Deviation and Skewness for each channel in HSV space have been used since most of the color distribution information is contained in the low-order moments. This results in a nine dimensional feature vector.

\section{Color Coherence Vector}

A color coherence vector (CCV) measures the spatial coherence of pixels with a given color by computing two color histograms: one for coherent pixels and another for incoherent pixels. It captures the details that a pixel is part of a coherent region or not. We have chosen Color Coherence Vector keeping in mind that natural scenes tend to have larger regions of similar color occupying a considerable part of the image as compared to urban scenes. This paper uses a fifty four dimensional CCV feature vector (discretization performed for 27 colors).

\section{Color Correlogram}

A color correlogram expresses how the spatial correlation of pair of colors changes with distance. Generally a correlogram for an image is a table indexed by color pairs, where the $\mathrm{k}^{\text {th }}$ entry for the row specifies the probability of finding a pixel of color $j$ at a distance $k$ from a pixel of color $i$ in the image. Extracting color correlogram for varying distances is computationally intensive. This paper therefore extracts color autocorrelogram which captures the spatial correlation between identical colors and thus reduces the feature dimension resulting in sixty four dimensional feature vector. The choice of color correlogram is guided by the fact that not only color component but its spatial location is also important in analyzing various scene categories particularly natural scenes. For example blue color at the top of the image indicates sky whereas blue region at the bottom of the image indicates the presence of water.

\section{Texture}

Texture plays an important role in domain-specific applications such as scene categorization. Some approaches [2] have used only texture orientation as a low level feature to discriminate 'city/suburb' images. The texture features used in our system are:

\section{GLCM}

Gray level co-occurrence matrices (GLCM) are generated by counting the number of occurrences of gray levels at a given displacement and angle. For a displacement value 2 (pixels at offset 2 ) and 4 angles [ $0^{\circ}$, $\left.45^{\circ}, 90^{\circ}, 135^{\circ}\right]$ statistics such as energy, contrast, correlation, homogeneity, variance and entropy are computed from the GLCM to obtain texture features as proposed by Haralick et al. in [19]. Correlation measures linear dependency of gray levels among neighborhood pixels and contrast measures the local variations. Homogeneity evaluates texture uniformity; entropy helps to analyze the randomness in texture whereas energy calculates local homogeneity. A twenty-four dimensional feature vector is used for detailed texture analysis.

\section{Gabor Filters}

Gabor filters are parameterized functions useful for analyzing textured patterns. They efficiently represent different image regions since they scale, rotation and displacement invariant. A family of self similar Gabor wavelets closely models the simple cells of visual cortex making it a suitable choice for our application. Our experimental setup creates a Gabor filter bank for 4 scales and 6 orientations (window size 39x39) resulting in total 
24 filters. Fig. 1 shows the magnitudes of Gabor filters. Fig. 2 shows the real parts of Gabor filters.

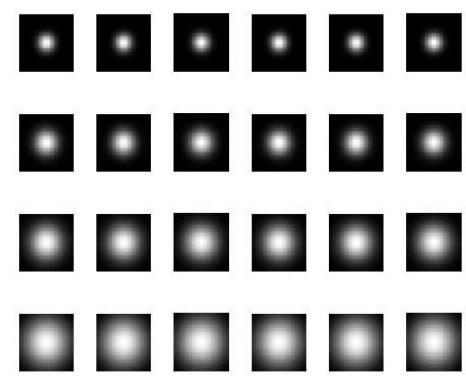

Fig.1. Magnitudes of Gabor Filters

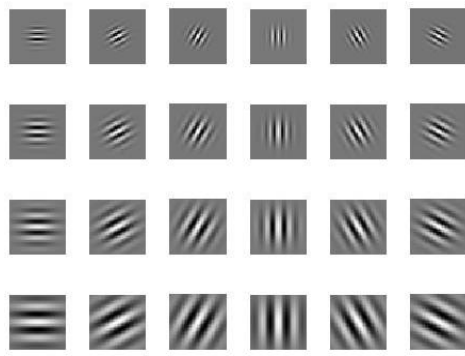

Fig.2. Real parts of Gabor Filters

For each scale and orientation, mean and standard deviation of magnitudes of transformed coefficients are calculated resulting in forty-eight dimensional feature vector. Figure 3 and Figure 4 show how an exemplar image is filtered using Gabor filter bank.

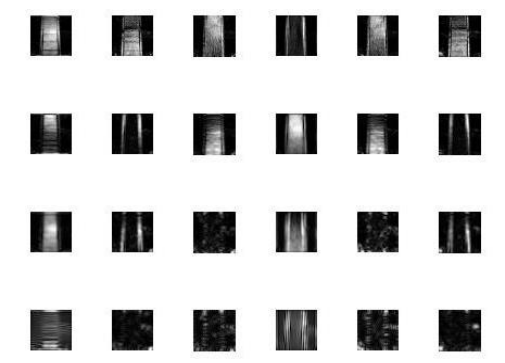

Fig.3. Magnitudes of Gabor Filtered Image

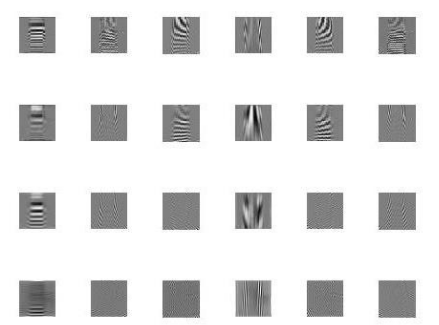

Fig.4. Real parts of Gabor Filtered Image

\section{Daubechies Wavelets}

Wavelet Transform decomposes the image into a series of high pass and low pass bands and extracts directional details that capture horizontal $(\mathrm{cH})$, vertical $(\mathrm{cV})$ and the diagonal (cD) activity. Since lower spatial frequencies of an image are more significant for the image's characteristics than higher spatial frequencies, further filtering of the approximation is useful. We use DB4 wavelets with three level decomposition (as shown in Fig. $5)$ similar to the approach used by authors in $[3,4]$.

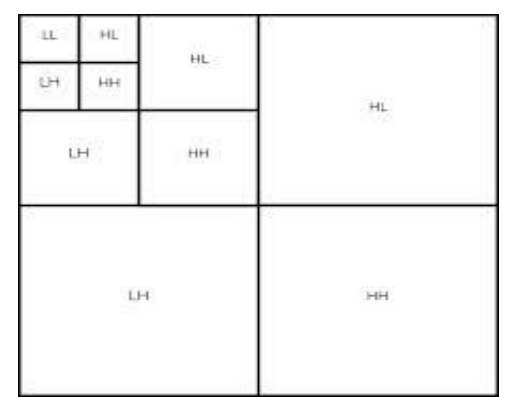

Fig.5. Daubechies three level decomposition

At each level of decomposition for HL, $\mathrm{LH}$ and $\mathrm{HH}$ sub-bands we calculate energy, mean and standard deviation resulting in a twenty-seven dimensional feature vector. Fig. 6 shows decomposition of an image using DB4 three-level decomposition. Fig. 7 shows the DB4 reconstructed image.
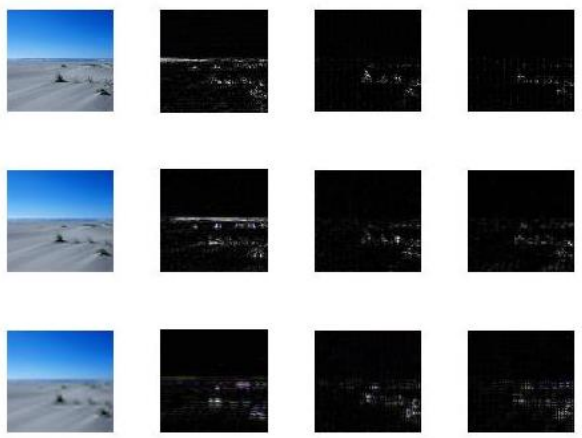

Fig.6. DB4 Three Level Decomposition of example image
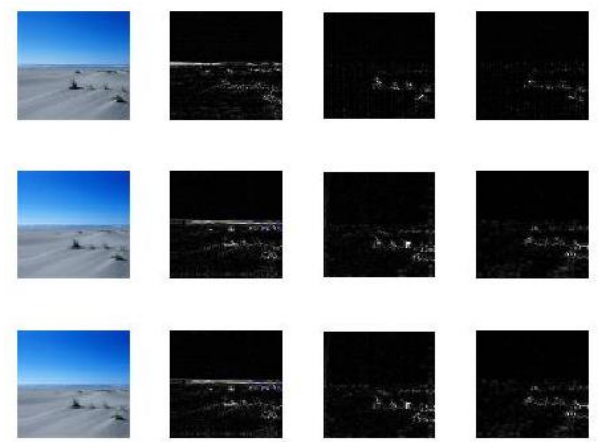

Fig.7. Reconstructed example image

Edges

In scene applications edges are dominant features. For example sky scrapers will have more vertical edges whereas mountain peaks will have prominent diagonal edges. To extract edge features we have used Edge Direction Histogram.

\section{Edge Direction Histogram}


The basic idea of Edge Direction Histogram is to build a histogram with the directions of the gradients of the edges (borders or contours). For scene images we are interested in the detection of the directions (angles) in which different edges occur. Edge Direction Histogram is used to describe the distribution of the edge points in each direction and is calculated by counting the number of the pixels in each user-defined direction. In [5] edge orientation along with other features has been used for indoor - outdoor scene classification based on neural network learning. In this paper edges are first detected using canny edge detector and edge pixels are counted in five directions vertical, horizontal, two diagonals and non-directional (which do not belong to any other category) resulting in a five dimensional feature vector. Table 1 summarizes the feature set used in our experiments.

Table 1. Feature set used in our approach

\begin{tabular}{|c|c|c|}
\hline Feature & Description & Dimension \\
\hline $\begin{array}{l}\text { Color } \\
\text { Moments(CM) (Z- } \\
\text { score normalized) }\end{array}$ & $\begin{array}{lr}\text { Low order } \\
\text { moments mean, } \\
\text { standard } \\
\text { deviation and } \\
\text { skewness in } \\
\text { HSV space. }\end{array}$ & 9 \\
\hline $\begin{array}{l}\text { Color Coherence } \\
\text { Vector(CCV) } \\
\text { (Normalized by } \\
\text { number of image } \\
\text { pixels) }\end{array}$ & $\begin{array}{l}\text { Two } 27 \text { bin } \\
\text { histograms for } \\
\text { coherent and } \\
\text { non coherent } \\
\text { regions }\end{array}$ & 54 \\
\hline $\begin{array}{l}\text { Color Correlogram } \\
\text { (CC) (Implicitly in } \\
\text { the form of } \\
\text { probability) }\end{array}$ & $\begin{array}{l}\text { Auto- } \\
\text { correlogram at } \\
\text { unit distance }\end{array}$ & 64 \\
\hline $\begin{array}{l}\text { GLCM (Z-score } \\
\text { normalized) }\end{array}$ & $\begin{array}{l}\text { Six statistical } \\
\text { features are } \\
\text { calculated for } \\
\text { each offset- } \\
\text { angle pair } \\
\end{array}$ & 24 \\
\hline $\begin{array}{l}\text { Daubechies } \\
\text { Wavelets(DB4) (Z- } \\
\text { score normalized) }\end{array}$ & $\begin{array}{l}\text { Energy, mean } \\
\text { and standard } \\
\text { deviation of } \\
\text { coefficients } \\
\text { obtained using } \\
\text { three level } \\
\text { decomposition }\end{array}$ & 27 \\
\hline $\begin{array}{l}\text { Gabor (Z-score } \\
\text { normalized) }\end{array}$ & $\begin{array}{l}\text { Mean, standard } \\
\text { deviation of } \\
\text { image filtered } \\
\text { using } 24 \text { filters }\end{array}$ & 48 \\
\hline $\begin{array}{l}\text { Edge } \\
\text { orientation/directio } \\
\text { n } \quad \text { histogram } \\
\text { (normalized by } \\
\text { number of edge } \\
\text { pixels) }\end{array}$ & $\begin{array}{l}\text { Count of edge } \\
\text { pixels in } 5 \\
\text { directions }\end{array}$ & 5 \\
\hline
\end{tabular}

\section{B. Dataset used}

We have used the Oliva-Torralba dataset (OT) [16] which is a subset of the Corel database. The dataset description is given in Table 2. Experiment 1uses 1472 images from natural scene category (OT4 dataset). Examples are shown in Figures 8a-8p. Experiment 2 uses all 2688 images from eight categories (OT8 dataset).
Table 2. Category wise details of dataset used

\begin{tabular}{|c|c|c|}
\hline Broad category & Internal category & $\begin{array}{c}\text { Number of } \\
\text { images }\end{array}$ \\
\hline Natural & Coast & 360 \\
\hline & Forest & 328 \\
\hline & Mountain & 374 \\
\hline & Open Country & 410 \\
\hline Urban & Tall Building & 356 \\
\hline & Street & 292 \\
\hline & Inside City & 308 \\
\hline & Highway & 260 \\
\hline Total & & 2688 images \\
\hline
\end{tabular}

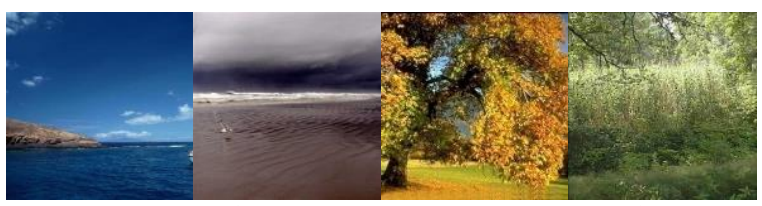
(a) Coast
(b) Coast
(c) Forest
(d) Forest
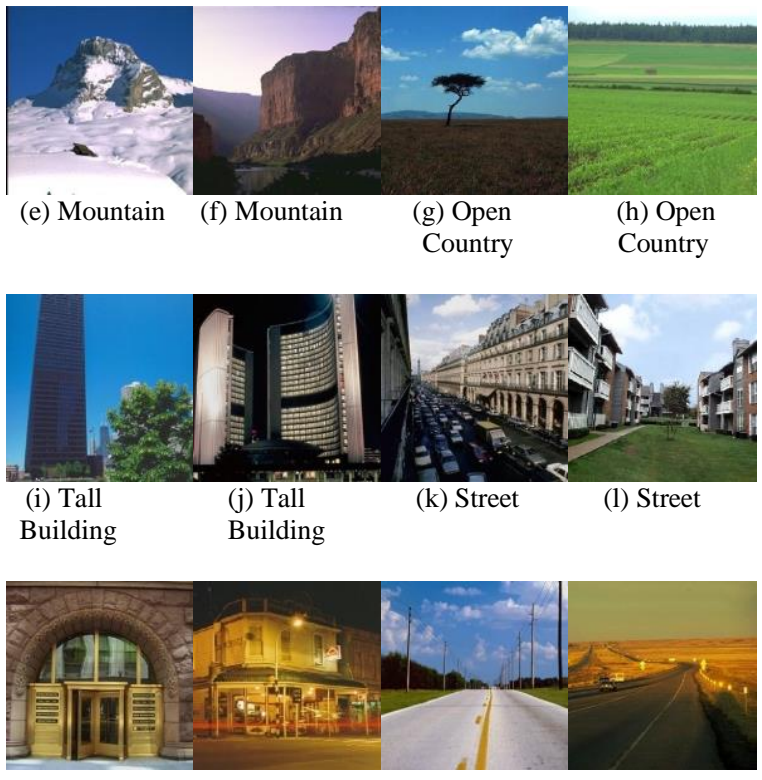

(m)Inside City (n)Inside City $\quad$ (o) Highway (p) Highway

Fig.8a-8p. Example images from each category.

\section{Feature Fusion}

Since no single feature can fully characterize an image we use feature fusion method with color, texture and edge features in this paper. Our system uses early fusion of feature vectors. Table 3 lists the fused feature combinations which have been evaluated for the two datasets in our experiments.

Table 3. Feature combinations generated after fusion

\begin{tabular}{|c|c|}
\hline Fused Feature Set & Feature Vector Dimension \\
\hline CM-Gabor-EDH & 62 \\
\hline CM-GLCM-EDH & 38 \\
\hline CM-DB-EDH & 41 \\
\hline CCV-Gabor-EDH & 107 \\
\hline CCV-GLCM-EDH & 83 \\
\hline CCV-DB-EDH & 86 \\
\hline CC-Gabor-EDH & 117 \\
\hline CC-GLCM-EDH & 93 \\
\hline CC-DB-EDH & 96 \\
\hline
\end{tabular}


$\mathrm{CM}$ is Color Moments, GLCM is Gray Level Co-occurrence Matrix, DB is Daubechies Wavelets, CCV is Color Coherence Vector, $\mathrm{CC}$ is Color Correlogram and $\mathrm{EDH}$ is Edge Direction Histogram.

\section{Classifier Design}

In this paper two discriminative models SVM and Artificial Neural Networks have been evaluated for scene classification.

\section{SVM Classifier}

Many authors have used SVM for scene classification. In [30] Gaussian kernel in an RBF-style classifier has been used. We have chosen SVM classifier because it is robust and effective even when less number of training samples is provided. This paper uses LIBSVM-3.20 package, dedicated for Matlab and have implemented One-Versus-All (OVA) technique for multi-class classification because it is faster, simpler and a complete approach. SVMs are parameterized by kernel function, box constraint constant $\mathrm{C}$ and a third constant depending on the type of kernel function used. The box constraint parameter $\mathrm{C}$ is the penalty parameter which controls the tradeoff between margin maximization and error minimization [25]. We have used Radial Basis Function (RBF) as kernel function therefore our SVM uses rbf_sigma as a parameter. For SVM tuning the training and test data has been normalized. Ten fold cross validation has been performed for automatic selection of $\mathrm{C}$ and $\gamma$ values. The best parameters are used to train SVM using whole training set.

\section{Artificial Neural Networks}

Neural networks belong to a family of statistical learning models inspired by biological neurons and have been used by authors for various classification problems.In [3] authors have used probabilistic neural network for classification of scenes into indoor/outdoor category. In [4] two neural classifiers: back propagation neural network and resilient back propagation neural network have been used on varying number of feature vectors corresponding to scene images tested on scene classification MIT database.

We have chosen neural networks due to their selfadaptive ability. We have used a two layer neural network. The hidden layer size is 10 . The training function used is 'trainscg' with learning rate set to 0.00001 and maximum number of epochs set to 5000 .

\section{EXPERIMENTAL RESULTS}

The goal of our experiments is to classify an unknown image as one of the learned scene classes. We perform two sets of experiments on an Intel Core i3 processor with 4GB RAM using MATLAB 2012a to analyze the different aspects of our model and learning approach. Two classifiers ANN and SVM have been implemented using nine fused feature combinations on OT4 and OT8 datasets. Tables 4 and 5 present the results of scene classification of OT4 dataset and Tables 6 and 7 present the results of scene classification of OT8 dataset by ANN and SVM respectively.

Table 4. Performance of OT4 dataset by ANN (Results obtained by cross validation)

\begin{tabular}{|c|c|c|c|c|c|c|}
\hline \multirow{2}{*}{$\begin{array}{c}\text { Fused Feature } \\
\text { Set }\end{array}$} & \multicolumn{5}{|c|}{ Fold Number } & \multirow{2}{*}{$\begin{array}{c}\text { Peak } \\
\text { Accuracy }\end{array}$} \\
\hline & 1 & 2 & 3 & 4 & 5 & \\
\hline $\begin{array}{l}\text { CM-Gabor- } \\
\text { EDH }\end{array}$ & 91.8 & 91.8 & 91.5 & 91.5 & 91.2 & 91.8 \\
\hline $\begin{array}{l}\text { CM-GLCM- } \\
\text { EDH }\end{array}$ & 82.3 & 82.3 & 82 & 81.6 & 81.3 & 82.3 \\
\hline CM-DB-EDH & 83.3 & 83.3 & 83 & 83 & 82.7 & 83.3 \\
\hline $\begin{array}{l}\text { CCV-Gabor- } \\
\text { EDH }\end{array}$ & 89.1 & 88.8 & 88.4 & 87.8 & 87.4 & 89.1 \\
\hline $\begin{array}{l}\text { CCV-GLCM- } \\
\text { EDH }\end{array}$ & 82.3 & 82 & 81.6 & 81.3 & 81 & 82.3 \\
\hline $\begin{array}{l}\text { CCV-DB- } \\
\text { EDH }\end{array}$ & 76.2 & 75.2 & 74.5 & 74.1 & 73.5 & 76.2 \\
\hline $\begin{array}{l}\text { CC-Gabor- } \\
\text { EDH }\end{array}$ & 74.1 & 71.7 & 71.1 & 70.7 & 70.1 & 74.1 \\
\hline $\begin{array}{l}\text { CC-GLCM- } \\
\text { EDH }\end{array}$ & 71.8 & 69.4 & 69 & 66.7 & 66.5 & 71.8 \\
\hline CC-DB-EDH & 74.8 & 73.8 & 73.5 & 73.1 & 72.8 & 74.8 \\
\hline
\end{tabular}

Table 5. Performance of OT4 dataset by SVM (Results obtained by cross validation)

\begin{tabular}{|c|c|c|c|}
\hline Fused Feature Set & $\mathrm{C}$ & $\Gamma$ & $\begin{array}{c}\text { Classification } \\
\text { Accuracy }\end{array}$ \\
\hline CM-Gabor-EDH & 3.668 & .016129 & $\mathbf{9 2 . 5}$ \\
\hline CM-GLCM-EDH & 663.9819 & .0012683 & 83 \\
\hline CM-DB-EDH & 5.6569 & .02439 & 83.7 \\
\hline CCV-Gabor-EDH & 663.9819 & .0010713 & 87 \\
\hline CCV-GLCM-EDH & 534.6682 & .001381 & 82.3 \\
\hline CCV-DB-EDH & 279.17 & .0020555 & 81.6 \\
\hline CC-Gabor-EDH & 20.7494 & .005422 & 86 \\
\hline CC-GLCM-EDH & 20.7494 & .0069723 & 77.5 \\
\hline CC-DB-EDH & 76.1093 & .0022868 & 79.2 \\
\hline
\end{tabular}

Table 6. Performance of OT8 dataset by ANN (Results obtained by cross validation)

\begin{tabular}{|c|c|c|c|c|c|c|}
\hline \multicolumn{6}{|c|}{$\begin{array}{l}\text { Classification accuracy of OT8 Dataset evaluation by } \\
\text { ANN }\end{array}$} & \multirow{2}{*}{$\begin{array}{l}\text { Peak } \\
\text { Perform } \\
\text { ance }\end{array}$} \\
\hline $\begin{array}{c}\text { Fused } \\
\text { Feature } \\
\text { Set }\end{array}$ & $\begin{array}{c}\text { Fold } \\
1\end{array}$ & $\begin{array}{l}\text { Fold } \\
2\end{array}$ & $\begin{array}{c}\text { Fold } \\
3\end{array}$ & $\begin{array}{c}\text { Fold } \\
4\end{array}$ & $\begin{array}{c}\text { Fold } \\
5\end{array}$ & \\
\hline $\begin{array}{c}\text { CM- } \\
\text { Gabor- } \\
\text { EDH }\end{array}$ & 85.1 & 84.5 & 84.2 & 84.2 & 84 & 85.1 \\
\hline $\begin{array}{c}\text { CM- } \\
\text { GLCM- } \\
\text { EDH }\end{array}$ & 81.6 & 80.4 & 80.3 & 79.9 & 79.7 & 81.6 \\
\hline $\begin{array}{l}\text { CM-DB- } \\
\text { EDH }\end{array}$ & 78 & 77.3 & 77.1 & 76.9 & 76.5 & 78 \\
\hline $\begin{array}{c}\text { CCV- } \\
\text { Gabor- } \\
\text { EDH }\end{array}$ & 81.2 & 80.6 & 80.1 & 79.9 & 79.7 & 81.2 \\
\hline $\begin{array}{c}\text { CCV- } \\
\text { GLCM- } \\
\text { EDH } \\
\end{array}$ & 76.9 & 76.2 & 75.2 & 74.7 & 74.3 & 76.9 \\
\hline $\begin{array}{c}\text { CCV- } \\
\text { DB-EDH }\end{array}$ & 73.1 & 72.9 & 72.5 & 72 & 71.7 & 73.1 \\
\hline $\begin{array}{c}\text { CC- } \\
\text { Gabor- } \\
\text { EDH }\end{array}$ & 72.8 & 71.9 & 71.7 & 71.3 & 70 & 72.8 \\
\hline $\begin{array}{c}\text { CC- } \\
\text { GLCM- } \\
\text { EDH }\end{array}$ & 62.6 & 61.6 & 61.1 & 58.8 & 55.7 & 62.6 \\
\hline $\begin{array}{l}\text { CC-DB- } \\
\text { EDH }\end{array}$ & 66.3 & 63.5 & 63.3 & 61.8 & 61.6 & 66.3 \\
\hline
\end{tabular}


Table 7. Performance of OT8 dataset by SVM (Results obtained by cross validation)

\begin{tabular}{|c|c|c|c|}
\hline Fused Feature Set & $\mathrm{C}$ & $\gamma$ & $\begin{array}{c}\text { Classification } \\
\text { Accuracy }\end{array}$ \\
\hline CM-Gabor-EDH & 20.7494 & .0043972 & $\mathbf{8 6 . 4}$ \\
\hline CM-GLCM-EDH & 663.9819 & .0018128 & 81.4 \\
\hline CM-DB-EDH & 430.539 & .0007622 & 78.4 \\
\hline CCV-Gabor-EDH & 181.0193 & .0020517 & 84.7 \\
\hline CCV-GLCM-EDH & 430.539 & .0050656 & 77.4 \\
\hline CCV-DB-EDH & 663.9819 & .00086425 & 76.5 \\
\hline CC-Gabor-EDH & 5.6569 & .020328 & 81 \\
\hline CC-GLCM-EDH & 76.1093 & .0045209 & 70 \\
\hline CC-DB-EDH & 181.0193 & .00077422 & 69.4 \\
\hline
\end{tabular}

Table 8 presents the comparative results of classification using ANN and SVM.

Table 8. Comparative Results of ANN and SVM

\begin{tabular}{|c|c|c|c|}
\hline \multicolumn{2}{|c|}{ OT4 Dataset } & \multicolumn{2}{c|}{ OT8 Dataset } \\
\hline ANN & 91.8 & ANN & 85.1 \\
\hline SVM & $\mathbf{9 2 . 5}$ & SVM & $\mathbf{8 6 . 4}$ \\
\hline
\end{tabular}

Figures 9 -12 show the confusion matrix for best performances reported by SVM and ANN over OT4 and OT8 dataset. Classes shown as 1,2,3,4,5,6,7,8 represent Coast, Forest, Mountain, Open Country, Tall Building, Street, Inside City, Highway.

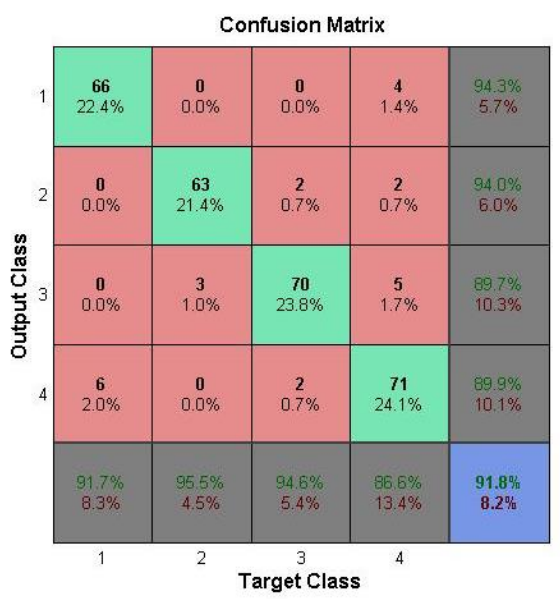

Fig.9. Confusion Matrix of classification of OT4 dataset by NN

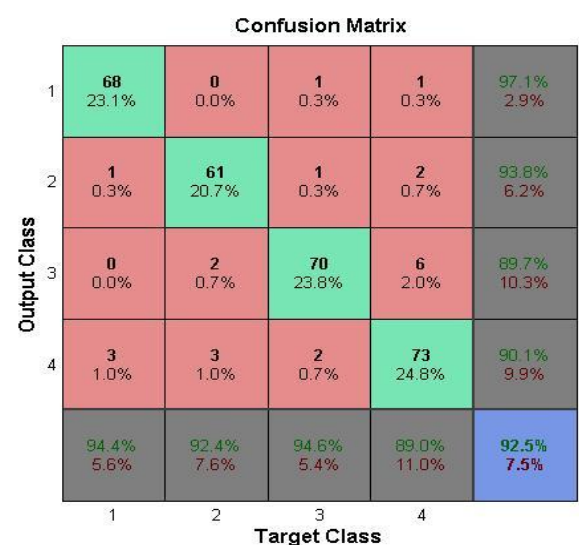

Fig.10. Confusion Matrix of classification of OT4 dataset by SVM

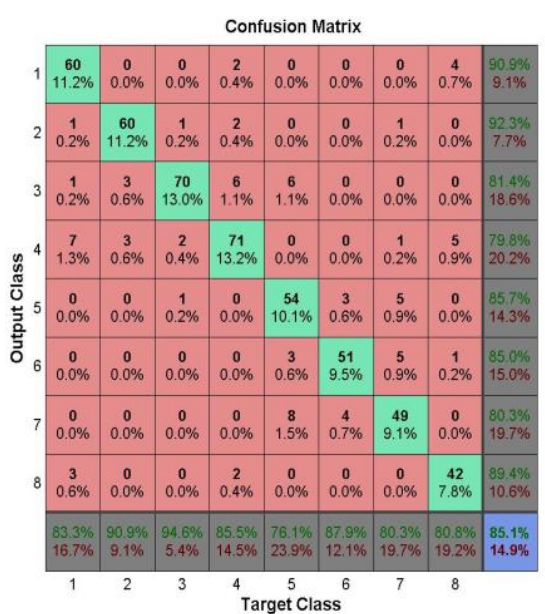

Fig.11. Confusion Matrix of classification of OT8 dataset by NN

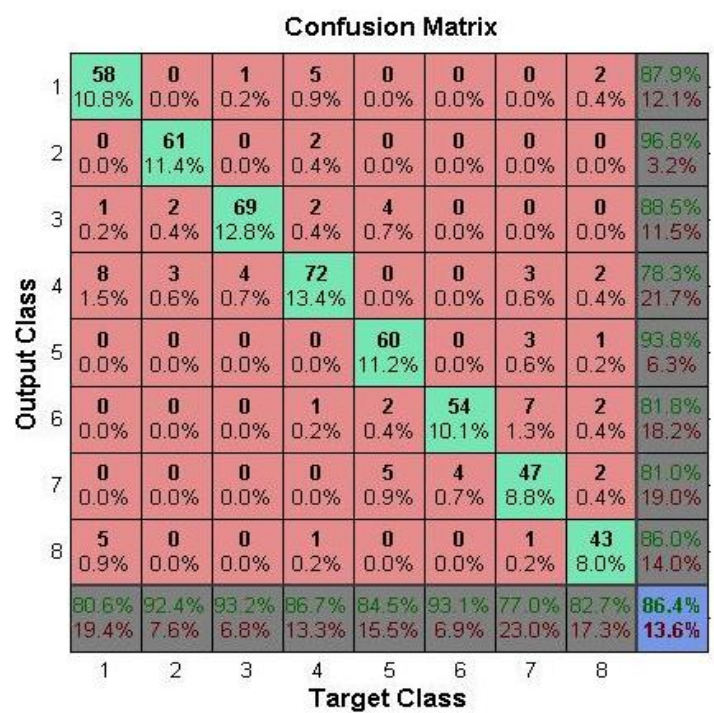

Fig.12. Confusion Matrix of classification of OT8 dataset by SVM.

Table 9. Predictive accuracy on OT8 Dataset

\begin{tabular}{|c|c|c|c|c|c|c|}
\hline \multirow{2}{*}{ Category } & \multicolumn{3}{|c|}{ SVM } & \multicolumn{3}{c|}{ NN } \\
\cline { 2 - 7 } & $\begin{array}{c}\text { Sensit } \\
\text { ivity }\end{array}$ & $\begin{array}{c}\text { Speci } \\
\text { ficity }\end{array}$ & $\begin{array}{c}\text { F- } \\
\text { score }\end{array}$ & $\begin{array}{c}\text { Sensiti } \\
\text { vity }\end{array}$ & $\begin{array}{c}\text { Specif } \\
\text { icity }\end{array}$ & $\begin{array}{c}\text { F- } \\
\text { score }\end{array}$ \\
\hline Coast & .8056 & .9828 & .8406 & .8333 & .9871 & .8696 \\
\hline Forest & .9242 & .9958 & .9457 & .9091 & .9894 & .9160 \\
\hline Mountain & .9324 & .9806 & .9079 & .9459 & .9654 & .8750 \\
\hline $\begin{array}{c}\text { Open } \\
\text { Country }\end{array}$ & .8675 & .9559 & .8229 & .8554 & .9604 & .8256 \\
\hline $\begin{array}{c}\text { Tall } \\
\text { Building }\end{array}$ & .8450 & .9914 & .8889 & .7606 & .9807 & .8060 \\
\hline Street & .9310 & .9749 & .8710 & .8793 & .9812 & .8644 \\
\hline $\begin{array}{c}\text { Inside } \\
\text { City }\end{array}$ & .7705 & .9769 & .7899 & .8033 & .9748 & .8033 \\
\hline Highway & .8269 & .9856 & .8431 & .8077 & .9897 & .8485 \\
\hline
\end{tabular}


Other than classification accuracy we also report sensitivity (fraction of positive patterns that are correctly classified), specificity (fraction of negative patterns that are correctly classified), f-measure (harmonic mean of precision and recall values) and kappa statistic (a measure of agreement between the machine learning classifier classifications and ground truth labels). Table 9, 10 show the predictive accuracy measures of classification by SVM and NN on OT8 and OT4 dataset respectively.

Table 10. Predictive accuracy on OT4 Dataset

\begin{tabular}{|c|c|c|c|c|c|c|}
\hline \multirow[b]{2}{*}{ Category } & \multicolumn{3}{|c|}{ SVM } & \multicolumn{3}{|c|}{ NN } \\
\hline & $\begin{array}{c}\text { Sens } \\
\text { itivit } \\
\mathrm{y}\end{array}$ & $\begin{array}{l}\text { Specifi } \\
\text { city }\end{array}$ & $\begin{array}{c}\text { F- } \\
\text { score }\end{array}$ & $\begin{array}{c}\text { Sensiti } \\
\text { vity }\end{array}$ & $\begin{array}{c}\text { Specific } \\
\text { ity }\end{array}$ & $\begin{array}{c}\text { F- } \\
\text { score }\end{array}$ \\
\hline Coast & $\begin{array}{c}.944 \\
4\end{array}$ & .9910 & .9577 & .9167 & .9820 & .9296 \\
\hline Forest & $\begin{array}{c}.924 \\
2\end{array}$ & .9825 & .9313 & .9545 & .9825 & .9474 \\
\hline Mountain & $\begin{array}{c}.945 \\
9 \\
\end{array}$ & .9636 & .9211 & .9459 & .9636 & .9211 \\
\hline $\begin{array}{c}\text { Open } \\
\text { Country }\end{array}$ & $\begin{array}{c}.890 \\
2\end{array}$ & .9623 & .8957 & .8659 & .9623 & .8820 \\
\hline
\end{tabular}

Table 11 reports the average accuracy measures.

Table 11. Average Performance Measures

\begin{tabular}{|c|c|c|c|c|}
\hline Predictive measure & \multicolumn{2}{|c|}{ Dataset OT4 } & \multicolumn{2}{c|}{ Dataset OT8 } \\
\hline & SVM & NN & SVM & NN \\
\hline Sensitivity & .9252 & .9184 & .8641 & .8510 \\
\hline Specificity & .9751 & .9728 & .9806 & .9787 \\
\hline F-score & .9252 & .9184 & .8641 & .8510 \\
\hline Kappa statistic & .899 & .8864 & .844 & .829 \\
\hline
\end{tabular}

\section{ANALYSIS OF RESULTS}

We have performed our experiments on two types of datasets- One which contains only natural scenes and the other which contains both natural as well as man-made structures. Many cases are very easily separable due to the different color, textural and/or structural composition. However the dataset used also contains overlapping classes. To solve this problem our aim was to find feature extraction methods that suited the domain and separated the classes better. State of the art feature extraction methods (color, texture and edge directionality features) and classification methods (Artificial Neural Networks and Support Vector Machine) were tested and the best of these were selected to be used in final scene classification system. We have used supervised learning algorithms for classification because they are able to learn with few images to facilitate the task of scene categorization. In order to evaluate the goodness of the implemented system a comparison between the results of the classifications and use of different feature sets has been presented in Section V. This section presents an analysis of the obtained results.

\section{A. Classifier Evaluation}

Specifically, to know the performance of the system a confusion matrix is computed. This is to check if the system is confusing two classes that is mislabeling an image of one class to another class. A confusion matrix shows the number of correct and incorrect predictions made by the classification model compared to the actual outcomes (target value) in the data. The overall performance rates are measured by the average value of the diagonal entries of the confusion matrix since they represent the number of correctly classified images. The off diagonal elements give us the false positives and the false negatives and thus represent the classification errors.

SVM performs better in both OT4 as well as OT8 datasets than Neural Networks in terms of classification accuracy. SVM gives peak classification accuracy of $92.5 \%$ for natural images in OT4 dataset and $86.4 \%$ for natural and urban images in OT8 dataset. The sensitivity and specificity values for SVM classifier for OT4 dataset as shown in Table 11 are .9252 and .9751 respectively. For OT8 dataset these values are .8641 and .9806 respectively. These high values indicate that the classifier is good at detecting the positives. At the same time SVM is capable of avoiding false alarms. Even though SVM performs better than $\mathrm{NN}$ in both datasets yet the performance can be greatly improved for OT8 dataset. We have also evaluated the kappa statistic to establish agreement between the expert and the classifier. In case of OT4 and OT8 dataset the kappa statistic value is .899 and .844 respectively. These high values indicate that the assignment of an image to a class is not random; rather the system has been well trained to classify the images. This shows the excellent classification ability of the classifier.

\section{B. Study of Misclassifications}

Figure 13 and 14 show the actual and predicted class (test images) for OT4 and OT8 datasets by SVM.
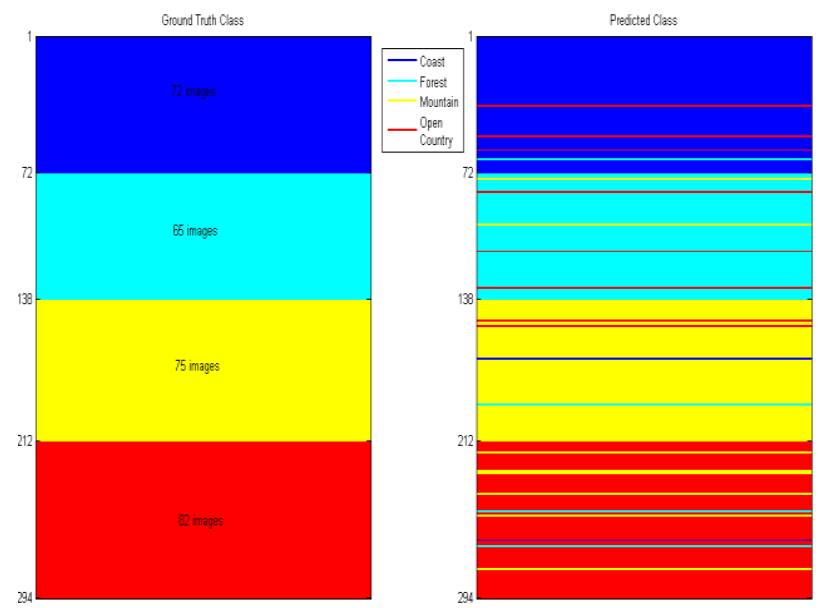

Fig.13. Actual versus predicted class OT4 dataset 


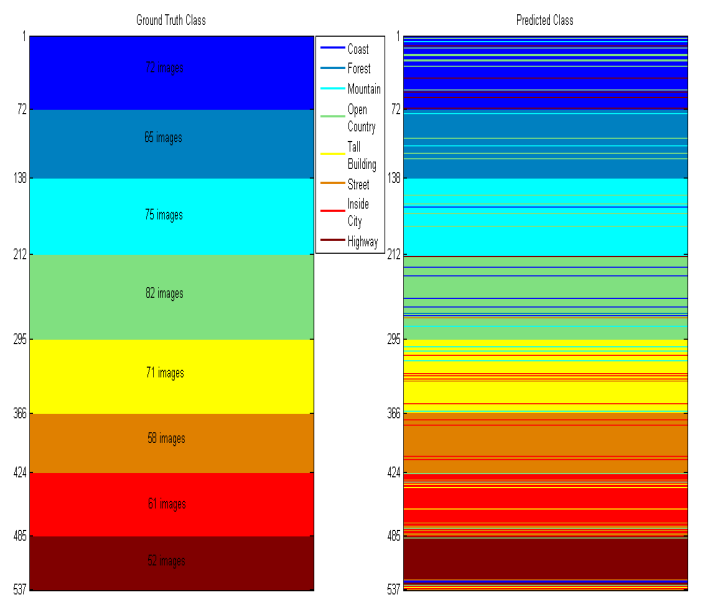

Fig.14. Actual versus predicted class OT8 dataset

In Figure 13 we can see that there are 72 test images of Coast class, 65 of Forest class, 75 of Mountain class and 82 of Open Country class.

Coast images are often misclassified as open country images (3/72). This is shown as red lines in the blue patch. There seems to be confusion between images from mountain and open country categories. 2/74 mountain images are classified as open country. It is also observed that open country images are misclassified as mountain images (6/82). This is shown as shown as yellow lines in the red patch.

The classifier is capable of distinguishing between coast and forest images. Only one coast images is classified as forest image (1/72). None of the forest images are classified as coast images. Forest images are misclassified as mountains and open country images may be due to occurrence of foliage making them semantically similar thus causing ambiguity.

As shown in Figure 14 Tall building images are classified into Inside City category (5/71). There also seems to be ambiguity between Street and Inside City images. 4/58 times street images are put into Inside City category and 7/61 times Inside City images are classified as street images. This may be due to the less expansiveness of both kinds of images. Highway images are misclassified as Street and Inside City images.

\section{B. Feature Evaluation}

The tests show that with the combination of best features, the accuracy of SVM is $92.5 \%$ and $86.4 \%$ for OT4 and OT8 dataset respectively.

In the case of different color features Color Moments produced good classification results than Color Coherence Vector and Auto Correlogram. Thus, among color features Color Moments are more discriminative. Among texture features the features extracted using Gabor filters are more discriminative than GLCM and Daubechies wavelets. The Edge direction histogram is discriminative as it identifies edges appropriately to distinguish between natural and urban images and also among categories of natural images.

\section{Performance Benefits of Selected Features}

Our system has achieved highest classification results with the combination of Color moments (color features), Gabor filters (texture features) and Edge histogram (edge distribution) since these features were compact within a category and distinct for different categories. We have performed feature level fusion which is advantageous because it utilizes the correlation between multiple features at an early stage which helps in better classification rate.

Lower order color moments are invariant to illumination and viewpoint and have efficiently interpreted the distribution of color in scene images. 2-D Gabor filters allow the study of the spatial distribution of texture. In the problem of scene classification Gabor filters have enabled the detection of gradual changes of texture and texture variations since the frequency and orientation representations of Gabor filters are similar to those of the human visual system. To capture rough global shape structure in an image edge direction histogram has been used which efficiently defines the distribution of direction of each edge pixel.

The comparative performance of various scene classification systems found in literature is given in Table 12. We have reported best classifier and feature combination of our work in this table.

Table 12. Performance Comparison

\begin{tabular}{|c|c|c|c|c|}
\hline Author & Approach & Features & Classes & Result \\
\hline $\begin{array}{c}\text { Our } \\
\text { Proposed } \\
\text { Method }\end{array}$ & SVM & $\begin{array}{c}\text { Color } \\
\text { Moments , } \\
\text { Gabor features, } \\
\text { Edge Direction } \\
\text { Histogram }\end{array}$ & 4 & 92.5 \\
\hline $\begin{array}{c}\text { Gupta et.al } \\
{[14]}\end{array}$ & $\begin{array}{c}\text { Foed- } \\
\text { Feurwal } \\
\text { Network }\end{array}$ & $\begin{array}{c}\text { Color Moments } \\
\text { (RGB), } \\
\text { Daubechies } \\
\text { wavelets }\end{array}$ & 3 & 82.66 \\
\hline $\begin{array}{c}\text { Grossberg } \\
\text { and }\end{array}$ & $\begin{array}{c}\text { Neural } \\
\text { system } \\
\text { Huang[22] }\end{array}$ & $\begin{array}{c}\text { Surface color } \\
\text { statistics, Three } \\
\text { principal } \\
\text { textures }\end{array}$ & 4 & 91.85 \\
\hline $\begin{array}{c}\text { Han and } \\
\text { Liu [7] }\end{array}$ & $\begin{array}{c}\text { Kernel PC } \\
\text { based } \\
\text { prototype } \\
\text { presentatio } \\
\text { n }\end{array}$ & $\begin{array}{c}\text { Color opponent } \\
\text { features, Spatial } \\
\text { layout of Gabor } \\
\text { features }\end{array}$ & 4 & 91.3 \\
\hline $\begin{array}{c}\text { Oliva and } \\
\text { [15] }\end{array}$ & $\begin{array}{c}\text { K-NN } \\
\text { classifier }\end{array}$ & $\begin{array}{c}\text { Naturalness, } \\
\text { Openness, } \\
\text { Roughness, } \\
\text { Ruggedness, } \\
\text { Expansion }\end{array}$ & 4 & 89 \\
\hline
\end{tabular}

\section{AdVANTAGES OF PROPOSED APPROACH}

This paper addresses the problem of scene classification without the need of segmentation and the processing of individual objects or regions thereby representing a scene image using a set of global image properties. The main aim of this work is to classify scenes using global low-level features. 


\section{Highlights}

Though different authors have attempted to derive global semantics from low-level features but the uniqueness of the approaches proposed in this paper are:

1) We propose a global-feature-based-system to derive semantics from images. The training and test images are not divided into sub-blocks / subimages which saves computation time and complexity and also results in reduced feature set derived holistically.

2) The proposed work does not rely on object recognition or object occurrences and their mutual relations as inaccurate segmentation can decrease categorization accuracy.

3) No involvement of human subjects like other approaches [15] to rank training images based on different properties (such as ruggedness, expansiveness and roughness etc.).

4) No requirement of classifying a large number of local patches [6] into semantic concepts (such as water, foliage, sky etc.) in order to train concept classifiers.

5) No semantic annotations or keywords of training images are required in the proposed system.

\section{CONCLUSIONS AND FUTURE DIRECTIONS}

The high classification accuracy achieved for natural images as well mixed images from natural and urban categories reveals that discriminative properties can be inferred from low-level image features. Low-level strategies for semantic scene classification have two clear advantages: their simplicity and their low computational cost. The classification of eight scene classes is complex since many images in different scene categories are very similar and ambiguous taking their semantic content into account. The class boundaries are not clear and in many cases the classes were not easily separable.

The major contribution of the work presented in this paper is successful semantic scene classification by the best combination of low-level features. After exhaustive experimentation we have found that the combination of holistic features (Color Moments-Gabor features-Edge direction histogram descriptors) proves to be the best in both SVM and Neural Network classifier. Increasingly, the research community has been pursuing the clear alternative of semantic features and we can derive midlevel features from the best combination of low-level features found in this work in order to reduce the "semantic gap". Intuitively we would also like to evaluate our method on other benchmarked datasets as well to check the effectiveness of the best features found so far. Our further research would be in two promising directions: Firstly to group a given collection of scenes into meaningful clusters according to the image content without a priori knowledge in an unsupervised manner allowing the system to extract meaningful patterns and association rules from clusters and secondly learning symbolic and / or semantic features from scenes using low-level features which should help bridge the semantic gap.

\section{REFERENCES}

[1] Ji Zhang, Wynne Hsu, Mong Li Lee, "Image Mining: Issues, Frameworks and Techniques", Proceedings of the Second International Workshop on Multimedia Data Mining (MDM/KDD'2001), in conjunction with ACM SIGKDD conference, San Francisco, USA, 26th August,2001.

[2] M. M. Gorkani and R. W. Picard, "Texture orientation for sorting photos at a glance", in Int. Conf. Pattern Recognition, Vol. 1, Oct. 1994, pp. 459-464.

[3] Lalit Gupta, Vinod Pathangay, Arpita Patra, A. Dyana and Sukhendu Das, "Indoor vs. Outdoor Scene Classification using Probabilistic Neural Network", EURASIP Journal on Advances in Signal Processing, Special Issue on Image Perception, Volume 2007, Issue 1, pp. 1-10.

[4] Amitabh Wahi, Sundaramurthy S., "Wavelet - Based Classification of Outdoor Natural Scenes by Resilient Neural Network", World Academy of Science, Engineering and Technology, International Journal of Computer, Control, Quantum and Information Engineering, Vol 8, No: 9, 2014.

[5] Li Tao, Yeong - Hwa Kim, Yeong - Taeg Kim, "An efficient neural network based indoor-outdoor scene classification algorithm", International Conference on Consumer Electronics (ICCE), Digest of Technical Papers(2010)., pp 317-318.

[6] J. Vogel and B. Schiele, "A semantic typicality measure for natural scene categorization", In DAGM'04 Annual Pattern Recognition Symposium, Tuebingen, Germany, 2004.

[7] Han, Y., Liu, G., "A Hierarchical GIST model embedding multiple biological feasibilities for scene classification", In Proc IAPR, Int. Conf. Pattern Recognition, Istanbul, (2012), pp. 3109-3112.

[8] A. Vailaya, A. Figueiredo, A. Jain, H. Zhang, "Image classification for content-based indexing", IEEE Transactions on Image Processing, Vol. 10, pp. 117-129, 2001.

[9] M. Szummer and R. Picard., "Indoor-outdoor image classification", IEEE International Workshop on Contentbased Access of Image and Video Databases, '98, Bombay, India, 1998.

[10] E.C.Yiu, "Image classification using color cues and texture orientation", Master's Thesis, Department of EECS, MIT (1996).

[11] I. Biederman, "On the semantics of a glance at a scene", in Perceptual Organizations, M. Kubovy and J. R. Pomerantz, Eds. Hillsdale, NJ: Lawrence Erlbaum, (1981), pp. 213-253.

[12] P. G. Schyns and A. Oliva, "From blobs to boundary edges: Evidence for time and spatial scale dependent scene recognition", Psychol. Sci., Vol. 5, (1994), pp. 195-200.

[13] E. Chang, K. Goh , G. Sychay, G. WU , CBSA, "Content based soft annotation for multimodal image retrieval using bayes point machines", IEEE Transactions on Circuits and Systems for Video Technology Special Issue on Conceptual and Dynamical Aspects of Multimedia Content Description, Vol. 13 (1) (2003), pp 26-38.

[14] Gupta, D., Singh, A.K., Kumari, D., Raina, "Hybrid feature based natural scene classification using neural network", International Journal of Computer Applications, (2012), pp 48-52, 41 (16). 
[15] A. Oliva, A. Torralba, "Modeling the shape of the scene: a holistic representation of the spatial envelope", International Journal of Computer Vision, Vol. 42 (3) (2001) pp 145-175.

[16] Aude Oliva, Antonio B. Torralba, Anne Guerin- Dugue and Jeanny Herault, "Global Semantic Classification of Scenes using Power Spectrum Templates ",Challenge of Image Retrieval (CIR99), Elect. Work. in Computing Series, Springer-Verlag, Newcastle, 1999.

[17] L.Fei-Fei, P. Perona, "A bayesian hierarchical model for learning natural scene categories", in: IEEE Computer Society Conference on Computer Vision and Pattern Recognition, Washington, DC, USA, (2005), pp. 524-531.

[18] A. Bosch, X. Munoz, A. Oliver, R Mart1', "Object and scene classification: What does a supervised approach provide us?", in: IAPR International Conference on pattern recognition, Hong Kong, 2006.

[19] R. M. Haralick, K. Shanmugam, and I. H. Dinstein, "Textural Features for Image Classification", IEEE Transactions on Systems, Man and Cybernetics, Vol. 3, no. 6,(1973) pp. 610-621.

[20] J Yu, D Tao, Y. Rui , J Cheng, "Pairwise constraints based multiview features fusion for scene classification", Pattern Recognition, Elsevier Volume 46 Issue 2, February (2013), pp. 483-496.

[21] Alessandro Perina, Marco Cristan, Vittorio Murino, "Learning natural scene classification by selective multiscale feature extraction", Image and Vision Computing, Elsevier, Volume 28, Issue 6, June (2010), pp. 927-939.

[22] S. Grossberg.T Huang, "ARTSCENE: A neural system for natural scene classification", Journal of Vision, (2009) Volume 9(4):6, pp. 1-19.

[23] S. Belongie, C. Carson, H. Greenspan, and J. Malik, "Recognition of images in large databases using a learning framework", (1997), Technical Report No. CSD 97-939, University of Califirnia Berkeley.

[24] Patricia G. Foschi, Deepak Kolippakkam, Huan Liu and Amit Mandvikar, "Feature Extraction for Image Mining", Proceedings in International workshop on Multimedia Information System, (2002), pp. 103-109.

[25] J.C. Burges, "A tutorial on support vector machines for pattern recognition", Data Mining Knowledge Discovery 2, (1998) 1-43.

[26] S. Paek, C.L. Sable, V. Hatzivassiloglou, A. Jaimes, B.H. Schiffman, S.-F. Chang, K.R. Mckeown, "Integration of visual and text based approaches for the content labeling and classification of photographs", ACM SIGIR '99 Workshop Multimedia Indexing Retrieval, Berkeley, CA, 1999.

[27] A. Savakis, J. Luo, "Indoor vs. outdoor classification of consumer photographs", Proceedings of IEEE International Conference on Image Processing, Thessaloniki, Greece, September 2001.

[28] A. Guerin-Dugue, A. Oliva, "Classification of scene photographs from local orientation features", Pattern Recognition Letters, 21 (2000), pp 1135-1140.

[29] N. Serrano, A. Savakis, J. Luo, "A computationally efficient approach to indoor/outdoor scene classification", International Conference on Pattern Recognition (2002), QuWebec City, Canada.

[30] Matthew Boutell, Anustup Choudhury, Jiebo Luo, Christopher M. Brown, "Using semantic features for scene classification: How good do they need to be?", IEEE International Conference on Multimedia and Expo, Toronto, July 2006.

[31] Ashoka Vanjare, Omkar S. N., J.Senthilnath, "Satellite Image Processing for Land Use and Land Cover
Mapping",Int. Journal of Image, Graphics and Signal Processing,2014,10,pp. 18-28.

\section{Authors' Profiles}

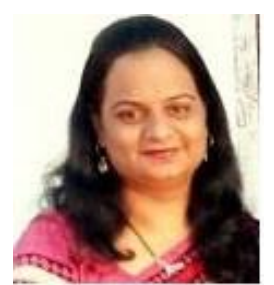

Mrs. Padmavati Shrivastava received her M.Tech. degree from Chhattisgarh Swami Vivekanand Technical University, Bhilai , Chhattisgarh, India , in 2011. She is presently a Ph.D. scholar in Dr. C.V. Raman University, Bilaspur Chhattisgarh, India. Her main research interests are in the area of Multimedia Data Mining, Information Retrieval, Computer Vision and Pattern Recognition. The author can be contacted at padmavati.shrivastava@yahoo.co.in

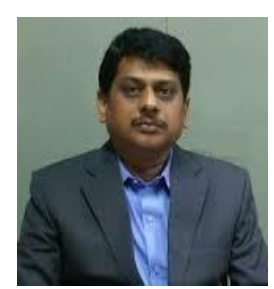

Dr. K.K. Bhoyar completed B.E. in Computer Science and Engineering in 1990 and M.Tech. in Computer Technology in 2001, both from SGGSIE\&T Nanded, an autonomous Institution funded by Govt. of India. He has been awarded Ph.D. degree in Computer Science \& Engg. from VNIT, Nagpur in July 2010, for his research work titled 'Performance Enhancement of Color Based Classification, and Segmentation for Image Retrieval using JND Approach.' He is presently working as Professor, in the Dept. of Information Technology at Yeshwantrao Chavan College of Engineering Nagpur (India). His areas of interests include Image Processing and Soft Computing.

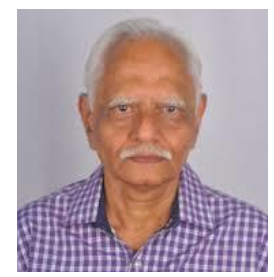

Dr. A. S. Zadgaonkar has obtained B. E. degree in Electrical Engineering from $\mathrm{Pt}$ Ravishankar Shukla University, Raipur in 1965 and M. E. in 1978 from Nagpur University. The testing technique for quality of wood developed by him is included in ISI in 1979. He was awarded Ph. D. in 1986 by Pt. Ravishankar Shukla University, Raipur on 'Investigation of Dynamic Properties of Non-Conducting Materials Using Electrical Analogy.' He has more than 47 years of teaching experience and has published more than 500 technical papers in various journals, and National and International conferences. He owes the credit of being the first Vice Chancellor of Dr. C. V. Raman University, Bilaspur (Chhattisgarh) and is presently serving as Advisor, Dr. C. V. Raman University, Bilaspur (Chhattisgarh). He is life member of Acoustical Society of India, Biomedical Society of India, Linguistic Society of India, Indian Society for Technical Education and many other social bodies. 